\title{
Proteins of the Nucleolus of Dictyostelium discoideum: Nucleolar Compartmentalization, Targeting Sequences, Protein Translocations and Binding Partners
}

\author{
Danton H. O'Day ${ }^{1,2}$ \\ 1 Department of Biology, University of Toronto Mississauga, Mississauga, ON L5L 1C6, Canada; \\ danton.oday@utoronto.ca; Tel.: +905-808-6566 \\ 2 Department of Cell and Systems Biology, University of Toronto, Toronto, ON M5S 3G5, Canada
}

Received: 30 January 2019; Accepted: 15 February 2019; Published: 17 February 2019

check for updates

\begin{abstract}
The nucleoli of Dictyostelium discoideum have a comparatively unique, non-canonical, localization adjacent to the inner nuclear membrane. The verified nucleolar proteins of this eukaryotic microbe are detailed while other potential proteins are introduced. Heat shock protein 32 (Hsp32), eukaryotic translation initiation factor 6 (eIF6), and tumour necrosis factor receptor-associated protein 1 (TRAP1) are essential for cell survival. NumA1, a breast cancer type 1 susceptibility protein-C Terminus domain-containing protein linked to cell cycle, functions in the regulation of nuclear number. The cell cycle checkpoint kinase 2 homologue forkhead-associated kinase A (FhkA) and BRG1-associated factor 60a homologue Snf12 are also discussed. While nucleoli appear homogeneous ultrastructurally, evidence for nucleolar subcompartments exists. Nucleolar localization sequences (NoLS) have been defined that target proteins to either the general nucleolar area or to a specific intranucleolar domain. Protein translocations during mitosis are protein-specific and support the multiple functions of the Dictyostelium nucleolus. To enrich the picture, binding partners of NumA1, the most well-characterized nucleolar protein, are examined: nucleolar $\mathrm{Ca}^{2+}$-binding protein $4 \mathrm{a}$ (CBP4a), nuclear puromycin-sensitive aminopeptidase A (PsaA) and Snf12. The role of Dictyostelium as a model for understanding the contribution of nucleolar proteins to various diseases and cellular stress is discussed throughout the review.
\end{abstract}

Keywords: nucleolus; Dictyostelium; nucleolar localization sequences; protein translocation; closed mitosis; disease

\section{Introduction}

The nucleolus is a multifunctional subnuclear compartment that has been studied for more than 200 years [1,2]. More than 4500 proteins comprise the human nucleolar proteome of which approximately $30 \%$ function in ribosome biogenesis. Historically, studies have primarily focused on the transcription of rDNA genes and rRNA processing leading to the assembly of ribosomal subunits that are exported to the cytoplasm. These functions underlie the basic structure and organization of eukaryotic bipartite or tripartite nucleoli. Tripartite nucleoli exhibit a fibrillar center (FC; transcriptionally inactive rDNA), a dense fibrillar component (DFC; transcriptionally active rDNA plus rRNA processing), and a granular component (GC; ribosomal subunit assembly). Bipartite nucleoli have overlapping FC and DFC plus a GC region. During mitosis the nucleolus disassembles during prophase and reassembles during telophase. Despite the early interest in ribosome biogenesis, about $70 \%$ of the nucleolar proteome functions in other events including cell signaling, centrosome function, chaperone activity, DNA replication and repair, molecular sequestration, regulation of cell cycle events, stress response regulation and viral replication $[3,4]$. This diversity of functions underlies the central role of the nucleolus in a diversity of human diseases [1,2]. 
Dictyostelium excels as a model biomedical research organism for a multitude of reasons. It is inexpensive and easy to culture with a one-day asexual, developmental life-cycle. Possessing a haploid genome facilitates the generation of mutants by a diversity of molecular techniques. These and other strains and vectors plus multiple other resources are available from the Dicty Stock Center at dictybase.org. The separation of growth and development with comparatively simple differentiation facilitate the study of many fundamental cellular processes including cell growth, cell death, cytokinesis, cell movement, chemotaxis, mitosis, phagocytosis, as well as morphogenesis and differentiation [5]. In the last decade or so, Dictyostelium has gained prominence for the study of cell stress as well as human diseases including Batten's disease, host-pathogen interactions, and Huntington's disease [6].

\section{The Dictyostelium Nucleolus}

As in other eukaryotes, the multiple nucleoli are the largest intranuclear bodies in Dictyostelium discoideum. Early research revealed that the structural features of this social amoebozoan's nucleoli differ from the classic nucleolar organization. Rather than localizing within the nucleoplasm, they exist as two to four dense patches that are tightly adhered to the inner nuclear envelope (Figure 1) [7-11]. What's more, they are neither bipartite nor tripartite. Instead, ultrastructurally they present as a more-or-less homogenous structure consisting of continuous fibrous matrices within which different-sized ribosome-like granules $(10-50 \mathrm{kDa})$ are distributed [12]. Fitting with the absence of defined FC regions, the rDNA instead forms a beaded ring-like structure (15-20 beads/ring) around the periphery of each nucleolus [13]. This rDNA is predominantly extrachromosomal with some being telomeric [5,14]. Other related amoebozoan species, including D. mucoroides, D. minutum, and Polysphondylium pallidum, appear to share the same nucleolar structure (e.g., [15,16]). In keeping with its role in rRNA synthesis in all species, treatment with actinomycin-D, an inhibitor of RNA polymerase I (i.e., rDNA transcription), leads to nucleolar breakdown in Dictyostelium [7,17].

This breakdown occurs in one of two ways: the progressive disappearance of protein localization (e.g., NumA1) or the formation of nucleolar buds-containing specific proteins (e.g., Cbp4a, Snf12, and FhkA)— that are released intact into the cytoplasm [18].
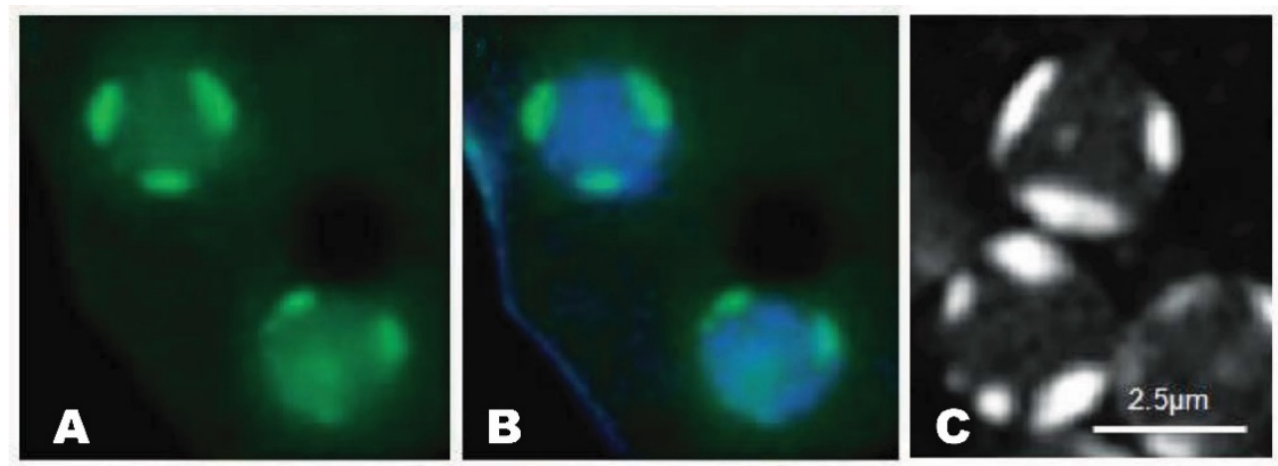

Figure 1. Nucleolar patches of Dictyostelium. A. GFP-NumA1 (green) localization. B. GFP-NumA1 localization with DNA (Hoescht, blue staining) stained. C. FITC-NumA1-NLS1 localization (white patches).

\section{Nucleolar Subcompartments in Dictyostelium}

Recent immunolocalization studies have shown that, despite earlier ultrastructural studies, the Dictyostelium nucleolus is not homogeneous (Figure 2) [18]. The nucleolar proteins of Dictyostelium organize as one of six observed patterns: localization to both the nucleolus and nucleoplasm (e.g., NumA1, eIF6, and Bud31), to the whole nucleolus (e.g., TRAP1) or to one of four subcompartments (NoSC1-4). CBP4a localizes to a patch close to the nuclear envelope designated as nucleolar subcompartment 1 (NoSC1). Snf12 localizes in NoSC2, a small speckle within NoSC1. 
The site of rDNA localization at the nucleolar periphery (NoSC3) coincides with general distribution of two nucleolar proteins, Hsp32 and FhkA. The localization of Src1, a helix-extension-helix family homolog, may be a nucleolar protein so until verified as one, subcompartment NoSC4 remains in question. This compartmentalization suggests there is more to the structure and function of the nucleus than has historically been recognized. The question remains as to whether each of these designated regions contain functionally related proteins.

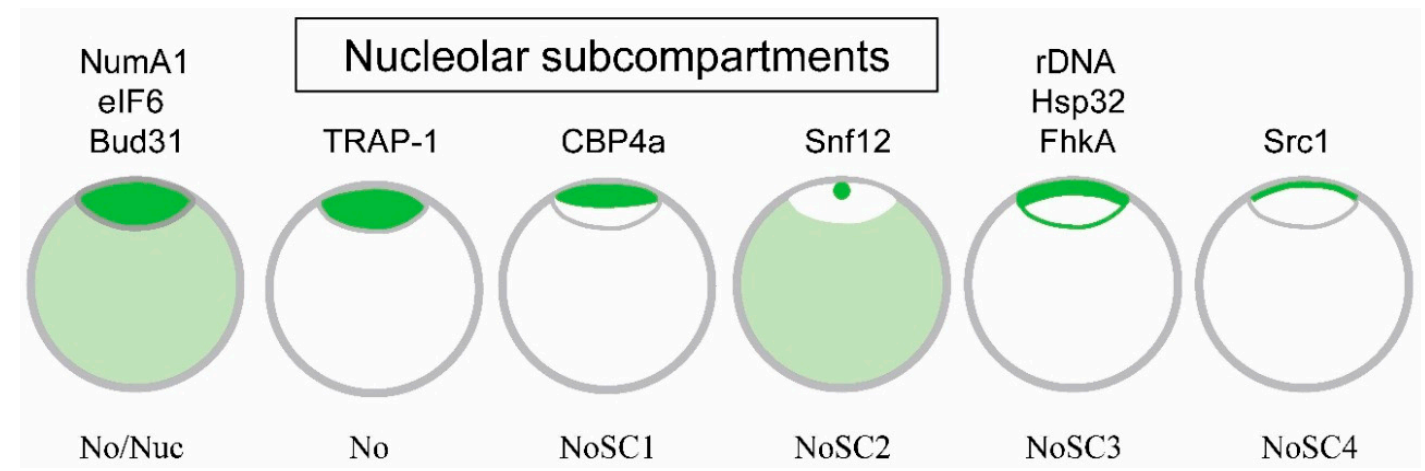

Figure 2. Localization of Dictyostelium nucleolar proteins. Different Dictyostelium nucleolar proteins localize differently as indicated by the green staining. The intensity of the staining summarizes their general differential localization in the nucleolus versus the nucleoplasm. NumA1, eIF6, and Bud31 localize to both the nucleolus and nucleoplasm (No/Nuc), TRAP-1 localizes only to the nucleolus (No), CBP4a localizes only to NoSC1, Snf12 localizes to NoSC2 as well as the nucleoplasm, while Hsp32 and FhkA localize to the nucleolar periphery, possibly representing NoSC3. Src1, a homolog of the helix-extension-helix family, is a questionable nucleolar protein that localizes to a region tentatively labelled NoSC4. The grey lines serve only to indicate the borders of the nucleolus and the nuclear envelope. (modified and updated after [18]).

The discovery of nucleolar subcompartments should permit researchers to define function-specific domains within the nucleolus to answer that question [18]. There are multiple sources of evidence that support the presence of nucleolar subcompartments in Dictyostelium. First, specific nucleolar proteins routinely localize to specific regions of the nucleolus (Figure 2). Treatment with actinomycin D results in two defined but distinct patterns of nucleolar protein departure: the loss of individual proteins that disperse through the nucleoplasm and/or cytoplasm (e.g., NumA1) and the formation of nucleolar buds enriched in specific proteins (e.g., CBP4a, Snf12, and FhkA). Finally, the NLS/NoLS from Snf12 (KRKR) specifically localizes GFP to NoSC2 and thus represents the first nucleolar subcompartment localization signal (NoSCLS) identified in Dictyostelium.

This is not to say the Dictyostelium nucleolus is a static region dominated by rigid subcompartments. Each of the nucleolar proteins shows different degrees of variability in their localization which fits with the work of others showing the size and shape of nucleoli change with varying conditions ]10]. However, the stage has been set to examine the significance, constancy and regulation of nucleolar subcompartmentalization. If we examine the general function of the nucleolar proteins that have been identified to date, the primary overlying theme is the general (e.g., NumA1, eIF1, Bud31) or localized (e.g., Cbp4a in NoSC1) distribution of proteins linked to cell cycle regulation. Two other proteins linked to cellular stress responses (i.e., Hsp32, FhkA) localize to nucleolar subcompartment NoSC 3 which could imply a localization of stress-related functions. Clearly, much remains to be done to prove the significance of the identified nucleolar subcompartments in Dictyostelium.

\section{Changes in Nucleolar Number and Positioning}

The nucleolus of Dictyostelium undergoes significant changes in shape, location and number in the transition from growth to development $[10,11]$. While 2-4 nucleoli characterize growth 
phase cells, this number diminishes to 1-2 during aggregation where one nucleolus resides in a nozzle-like nuclear protrusion that points in the direction of cell migration. With time only this single, microtubule-dependent, nozzle-localized nucleolus remains. These nucleolar events coincide with the turnover of $75 \%$ of the growth phase rRNA during development [19].

Nucleoli are excluded from the centrosomal region showing a preferential localization that is opposite to its location [10]. Nucleolar positioning as well as nucleolar size and number are directly linked to the level of ongoing ribosome production [20]. Clearly there are differences between growth phase and developmental nucleoli. A nucleolar mutant in Dictyostelium that only forms nucleoli during growth progressively loses its nucleolus during development but is unable to generate the developmental nucleolus [21]. This "anucleolate" mutant is unable to complete development but refeeding of the cells induces the reformation of growth phase nucleoli. The underlying reasons for this developmental deficiency remain to be studied but might indicate that an early evolutionary stage was the physical separation of interphase/mitotic nucleolar function from specific developmental nucleolar roles. Finally, nucleolar breakdown is one of the earliest events detected in the events of cell death associated with stalk cell differentiation in Dictyostelium suggesting that this event might be a common initial step in developmental cell death in other organisms [22].

\section{The Nucleolar Proteins of Dictyostelium}

In the following sections, each of the characterized nucleolar proteins is discussed in order of its discovery as a resident of the nucleolus. These short descriptions encapsulate the essence of each protein's role as a nucleolar protein. In each section, binding proteins, the presence of nucleolar localization signals and the translocations of the proteins during mitosis will be summarized as a prelude to summarizing the data and/or drawing broader conclusions about them. During mitosis in mammals, nucleolar proteins typically redistribute to other cellular locales upon dissolution of the nucleolus [2,23]. In Dictyostelium, which undergoes a semi-closed mitosis, the pattern of microtubule localization is typically used to determine mitotic stages $[9,24,25]$. To date, only five studies have been done on nucleolar protein translocations during mitosis in Dictyostelium.

\subsection{Heat Shock Protein 32 (Hsp32)}

The first "resident" nucleolar protein to be identified in Dictyostelium was heat shock protein 32 (Hsp32) [26]. Colocalizing with rDNA as beads on a string around the periphery of the nucleolus in unstressed cells, during heat shock it redistributes throughout the nucleolus and nucleoplasm $[13,26]$. Extended periods of heat shock produce a nucleolus with more pronounced rDNA beads revealing that the structure of the nucleolus in Dictyostelium responds to stress, as it does in other organisms [26,27]. In keeping with this idea, heat shock treatment of Dictyostelium induces a redistribution of Hsp32 from the nucleolar periphery and Snf12 from the nucleoplasm to nucleolus, as discussed below. Attempts to knock out the Hsp32 gene have failed suggesting it may be a critical protein [26].

Due to the presence of a highly acidic region rich in aspartic acid (asp) and glutamic acid (glu) residues, Hsp32 shares sequence similarity to nucleophosmin (NPM1) and nucleolin, both highly conserved nucleolar proteins in mammals [28]. The highly acidic regions are a common feature thought to be responsible for binding to basic ribosomal proteins and to NLSs of other proteins $[29,30]$. The role of the acid rich glu/asp region is discussed again for NumA1 below. Hsp32 also binds with high affinity to DNA but this association is not involved in its localization [26].

Hsp32 possesses a monopartite and bipartite NLS, thus sharing similarity with nucleolar proteins from other species [26,31,32]. However, an NoLS has not been identified and the means by which Hsp32 localizes to the nucleolus remains to be elucidated.

\subsection{Eukaryotic Translation Initiation Factor 6 (eIF6)}

Eukaryotic translation initiation factor 6, eIF6, was originally identified as a nucleolar protein based on its localization as peripheral patches in DAPI-stained nuclei plus its sensitivity of actinomycin 
D treatment). [33]. This highly conserved protein is essential to the production of 605 ribosomal subunits serving as a rate-limiting step in the cell cycle [2,34-36]. The pathogenesis of two forms of leukemia-inherited Shwachman-Diamond syndrome (SDS) and sporadic SDS-involve a common pathway in 60S-subunit maturation and the functional activation of ribosomes [37,38]. eIF6 is involved in 60S-subunit maturation and thus could play a central role in the disease process. Since eIF6 is shared by eukaryotes and archaea, Dictyostelium serves as a model to detail its function. For example, Weiss et al. [39] used single-particle cryo-EM to dissect the mechanism by which eIF6 gets released from nascent 60S ribosomal subunits in Dictyostelium. As in other species, eIF6 prevents 60S maturation by blocking the binding of essential maturation factors and, thus, must be removed for functional ribosomal formation to occur. This conserved mechanism involving eIF6 release is impaired in both inherited and sporadic leukemias.

The N-terminal region of eIF6 contains both an NLS and NoLS with a second potential NLS in the C-term [33]. Scott et al. [40] published an "Experimentally determined NoLS" and a predicted NoLS for eIF6, the latter generated from a program they compiled. However, based on their information summarized in Table 2 of their publication, they erroneously analyzed the sequence of NumA1 not eIf6, albeit with additional errors. Using their program to analyze eIF6 in fact detects no NoLS for this nucleolar protein (O'Day, unpublished results). So, although the NoLS in eIF6 has been mapped to a subdomain, the precise location of the NoLS for eIF6 remains to be revealed. Deletion of the eIF6 gene is lethal in Dictyostelium as it is in other species [34-36]. Examination of the data from Sillo et al. [41], from their study of genes linked to phagocytosis, revealed that eIF6 is upregulated by factors that induce phagocytosis in Dictyostelium.

\subsection{Tumor Necrosis Factor Receptor-Associated Protein 1 (TRAP1)}

Tumor necrosis factor receptor-associated protein 1 (TRAP1) is a member of the Hsp90 family [42,43]. It is a multifunctional protein linked to cell cycle progression, cell differentiation, and apoptosis. Found in the outermost layer of the spore coat it is believed to protect these dormant structures from physicochemical stresses [44,45]. While TRAP1 is a questionable nucleolar protein, there is some evidence it localizes there as well as in other cellular locations including mitochondria to where it translocates during early differentiation [45]. It was first observed in intranuclear patches reminiscent of nucleoli but not verified through actinomycin D or other treatments. As expected, TRAP1expression is induced by heat shock and like other members of the Hsp90 family null mutations are lethal [42,43]. More recently, TRAP1 has gained attention as a protein that can help with understanding mitochondrial diseases [46].

\subsection{Nucleomorphin A1 (NumA1)}

The nucleolar localization of a group of well-established calmodulin-binding proteins from other organisms such as calcineurin, CaM kinase II, and myosin light chain kinase supports a role for calmodulin in the nucleolus [47,48]. In keeping with this, this calcium-sensor and -effector has also been shown to bind and localize in mammalian nucleoli $[49,50]$. Nucleomorphin isoform NumA1 represents the only verified nucleolar calmodulin-binding protein in Dictyostelium. Acting as a regulator of nuclear number and interacting with calmodulin in a $\mathrm{Ca}^{2+}$-dependent manner, it is predominantly a nucleolar protein with secondary nucleoplasmic localization [51-53]. This pattern of localization is a common feature of nucleolar proteins (e.g., nucleophosmin, adenosine deaminases, and murine double minute 2) that shuttle between the nucleolus and nucleoplasm [54,55].

Full length nucleomorphin contains a breast cancer carboxy-terminus domain (BRCT) that is found in cell cycle checkpoint proteins in other organisms $[51,56]$. The presence of a highly acidic, glu/asp domain is a common feature of nucleolar proteins including nucleophosmin, nucleoplasmin, nucleolin, and Hsp32 [26,28]. Overexpression of GFP-NumA1 lacking its palindromic glu/asp or DEED domain results in multinuclearity fitting with NumA1's involvement in cell cycle regulation [51]. In contrast to the regulation of nuclear number in human cells where over 100 proteins appear to be involved, this is the only protein so far linked to this function in Dictyostelium [20]. 
A large number of attributes of NumA1 suggest it is a functional equivalent of the mammalian nucleolar protein nucleophosmin (NPM1) [51]. NPM1 has diverse functions including a role in DNA repair, centrosome duplication and cell proliferation. Mutations in NPM1 are a major cause of acute myeloid leukemia (AML) being present in $20-30 \%$ of the cases [57]. The acidic glu/asp domain of NPM1 is involved in histone binding but this function has not been studied in NumA1. The DEED domain of NumA1 is sufficient to target FITC to the nucleus thus acting as an unconventional NLS [51]. Furthermore, NPM1 can act as both a proto-oncogene and as a tumor suppressor [58]. The study of NumA1 thus has the possibility of offering additional insight into the mode of action of NPM1. For example, as covered in the next section, its DEED domain binds Cbp4a in a calcium-dependent manner suggesting NumA1 could be involved in recruiting other proteins to the nucleolus, a mechanism apparently not yet studied for NPM1.

Yeast two hybrid studies revealed that NumA1 interacts with the calcium-binding protein Cbp4a and $\mathrm{Zn}^{2+}$-metallopeptidase puromycin-sensitive aminopeptidase A (PsaA) which, in other species, is associated with cell cycle progression and several human diseases including Huntington's and Alzheimer's disease [59]. Dictyostelium PsaA is similar to PSA from Drosophila, mouse, and human. In contrast to Cbp4a (see below), PsaA does not localize to the nucleolus but colocalizes with NumA1 in the nucleoplasm independent of $\mathrm{Ca}^{2+} /$ Calmodulin [60]. The functional relationship between Cbp4a and NumA1 is strengthened by their apparent co-regulation by developmental morphogens where differentiation factor-1 (DIF-1) upregulates them while cyclic AMP and ammonia leads to their downregulation [61].

An attribute of some nucleolar proteins is the presence of multiple NLSs. NumA1 contains four identified NLSs [51,62]. Three of them (NLS-1, -2, and -4) reside within N-terminal residues 1-120: NLS1, ${ }^{31}$ PKSKKKF ${ }^{37}$ and NLS-1 and -4 within ${ }^{48}$ KKSYQDPEIIAHSRPRK $^{64}$ (NLS-4 is underlined). NLS-3 is found in the C-terminus ( ${ }^{246}$ PTKKRSL ${ }^{252}$ ). Multiple NLSs may function to modulate the amount of the nuclear-localized protein, as seen for human TBX5, nucleolar ribosomal protein L7a and transcription factor Nrf2 [63-65]. FITC-peptide constructs showed each of these sequences, including the terminal RPRK sequence of the bipartite NLS (48-64), localize to nucleoli revealing the peptide sequences are all NoLS/NLS (e.g., Figure 1) [60]. As with the localization of GFP-NumA1 the nucleolar localization was abolished in the presence of actinomycin D but unaffected by treatments with calcium chelators or calmodulin antagonists. Hence these sequences serve as joint NLS/NoLS for the targeting of NumA1. NoLSs, like NLSs, are typically rich in basic residues but, in the absence of a nucleolar envelope, rather than serving as transport signals they appear to function more as retention signals [66]. Human proteins containing NLS/NoLSs include human NF-KB-inducing kinase, the novel human nucleolar protein phosphatidylinositol 4-kinase and others [66,67].

In addition to NumA1, mitotic translocations have only been studied for four other proteins-Cbp4a, Src1, Snf12 and FhkA—as discussed in the following sections (Figure 3). During prophase nucleoli become indistinct with NumA1 appearing in smaller accumulations adjacent to the inner nuclear envelope as well as being associated with the centrosome. By metaphase these inner membrane accumulations disappear and, for the rest of mitosis, NumA1 appears throughout the cytoplasm with a border of protein adjacent the outer nuclear envelope plus continued centrosomal localization.

\subsection{Calcium Binding Protein 4a (Cbp4a)}

Studies using yeast two hybrid and coimmunoprecipitation, identified calcium binding protein $4 \mathrm{a},(\mathrm{Cbp} 4 \mathrm{a})$ as a nucleolar $\mathrm{Ca}^{2+}$-dependent, NumA1-binding partner [52]. This binding occurs via the glu/asp or DEED domain. Addition of actinomycin D leads to a loss of nucleolar CBP4a as does calcium-chelation with BAPTA-AM, supporting Cbp4a as a nucleolar protein that requires calcium for its localization. Fitting with its association with the cell cycle protein NumA1, CBP4a has a putative forkhead-associated domain that is present in numerous cell cycle proteins. Dictyostelium possesses 
13 calcium-binding proteins (CBPs) including CaM, a major $\mathrm{Ca}^{2+}$ effector in all eukaryotes [68-70]. In contrast to CaM, calfumirin, and CBP3, the function of the other CBPs remains to be researched [53].

Cbp4a residues ${ }^{40} \mathrm{KKCK}^{43}$ have been verified as a true NLS but not an NoLS since FITC-bound peptides show nuclear but not nucleolar localization. In total the results indicate that Cbp4a localizes to the nucleolus not via an NoLS but via the calcium-dependent binding to the DEED domain of NumA [60]. This would suggest that Cbp4a diffuses into the nucleolus to be held there through NumA1-binding. The relationship and behavioral differences between these two proteins was revealed during the events of mitosis where they were the first two nucleolar proteins to be studied during mitosis in Dictyostelium.

During mitosis CBP4a reveals a unique distribution that suggests the presence of previously undetected intranuclear subdomains that persist throughout the mitotic stages (Figure 3) [71]. During prophase, nucleolar dissolution is accompanied by the accumulation of CBP4a as multiple, discrete nucleoplasmic accumulations called "CBP4a islands". In addition to these multiple smaller accumulations, during metaphase two larger islands localize to the metaphase plate region. Through anaphase and telophase, these accumulations migrate to the inner membrane as if in anticipation of reforming post-mitotic nucleoli. To date, no other nucleolar or nuclear protein has shown this sequence of events during mitosis. If the nucleolar binding of $\mathrm{Cbp} 4 \mathrm{a}$ is dependent on diffusion, then retaining this protein within the nucleus may be essential to ensuring there is enough protein present when the nucleolus reforms during telophase.

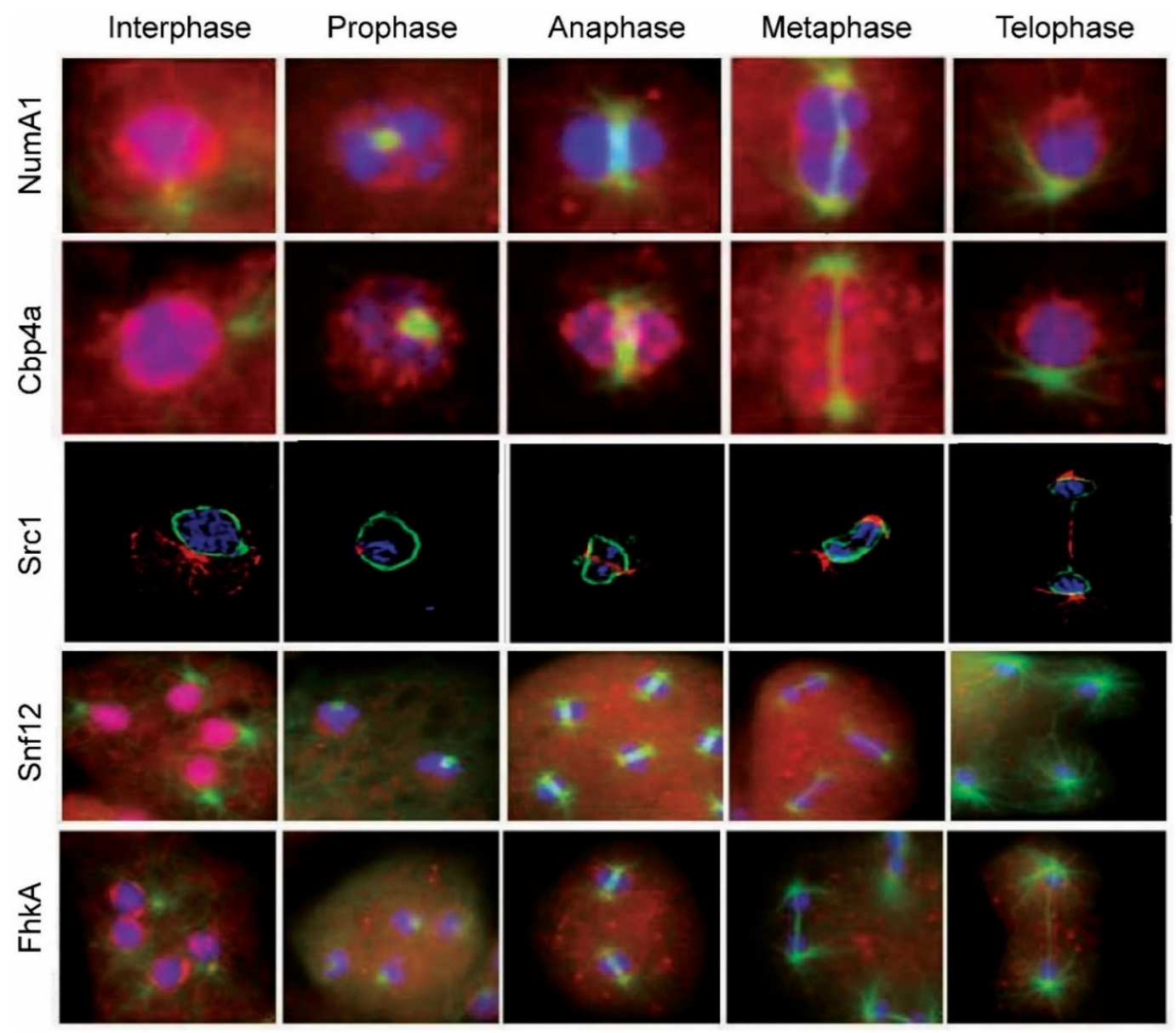

Figure 3. Nucleolar protein translocations during mitosis in Dictyostelium discoideum. NumA1, nucleomorphin A1, a cell cycle protein; $\mathrm{Cbp} 4 \mathrm{a}$, calcium-binding protein $4 \mathrm{a}$, a NumaA1 binding protein; Src1, helix-extension-helix family homolog; Snf12, a nucleosome remodeling complex component; FhkA, a Rad53 (Chk2 in humans) tumor suppressor homolog. Note: the images for Src1 are from [72] with modifications. 


\subsection{SWI/SNF Complex Component SNF12 Homolog (Snf12)}

SWI/SNF is a nucleosome remodeling complex, composed of 9-12 proteins called BAFs (Barrier-to-autointegration factor), highly conserve proteins that regulate gene transcription [73]. The complex mediates multiple other processes including cell proliferation, differentiation and DNA repair. It serves as a tumor suppressor by regulating the p53-mediated transcription of cell cycle genes. BAF60a (Snf12 in yeast) mediates its interaction with p53. Dictyostelium Snf12 is a predominately nucleoplasmic protein that localizes to nucleoli in $\sim 20 \%$ of cells, as seen in mice $[74,75]$. Appropriately, it possesses conserved SWIB and COG domains found in BAF proteins, but these are not involved in nucleolar positioning. Instead an experimentally defined NLS/NoLS $\left({ }^{372} \mathrm{KRKR}^{375}\right)$ defines both its nuclear and nucleolar localization.

Unexpectedly, treatment of cells with actinomycin D increases the levels of nucleolar Snf12 which leads to an outward bulging of nucleoli followed by the cytoplasmic accumulation of Snf12-rich vesicles. Heat shock treatment also leads to a major increase in the nucleolar localization of Snf12. The rapid increases in nucleolar localization after heat shock and actinomycin D treatment, suggests Snf12 may function in the stress response. As mentioned above, heat shock treatment of Dictyostelium induces a redistribution of Hsp32 from the nucleolar periphery [26]. This is not surprising since the nucleolus is the central hub for coordinating the response to cell stress in other species where the composition of the nucleolus is stress-dependent [76]. In keeping with the results summarized here, heat shock and AM-D treatment both cause nucleolar accumulation of specific human proteins [77].

Snf12 undergoes several translocations during mitosis (Figure 3). With nucleolar dissolution during prometaphase, it first shifts from its nucleolar locale to take up a predominantly nucleoplasmic location with some localization in the cytoplasm. During metaphase and through anaphase it then exhibits a relatively uniform cellular distribution before reacquiring its nuclear/nucleolar localization during telophase.

\subsection{Forkhead-Associated Kinase Protein A (FhkA)}

Rad53 (CHK2 in humans) is a tumor suppressor protein involved in DNA damage (genotoxic) stress response [78]. It is recognized as a nuclear protein that possesses a C-terminal bipartite NLS [79]. The Dictyostelium Rad53 homologue forkhead-associated kinase protein A, FhkA, is a nucleolar protein. Immunolocalization shows is resides at the periphery of the nucleolar patches (i.e., NoSC3) being more concentrated adjacent to the nuclear envelope. Like Snf12, actinomycin D treatment leads to its nuclear expulsion as nucleolar protein-containing vesicles that end up in the cytoplasm. Its mitotic dynamics are also fitting for a nucleolar protein, yet its specific function there remains to be elucidated. During mitosis FhkA redistributes throughout the cell with an enhanced level of localization evident adjacent to the nuclear envelope from prometaphase through telophase (Figure 3). Like NumA1, FhkA also localizes within the spindle fiber region.

\subsection{Bud31}

As one of the last nucleolar proteins to be identified so far, less is known about Bud31 than any of the others. A comparative study of spliceosomal genes in Dictyostelium discoideum identified Bud31 but no further analysis of the protein was carried out [80]. In yeast where the protein was first identified, Bud31 is involved in cell cycle regulation, specifically functioning at the G1/S regulatory or start point [81]. A search of dictyBase.org indicates Bud31 is a putative RNA splicing factor or transcription factor. While the gene has been identified in humans and other species, its function in Dictyostelium has not been studied. Selected as a nuclear protein for comparison, it was shown that Bud31 localized throughout the Dictyostelium nucleolus along with NumA1 and eIF6 (Figure 2) [18]. However, its location during mitosis and the presence of an NoLS have not been assessed. 


\section{9. $\operatorname{Src1}$}

Dictyostelium Src1 is homolog of the helix-extension-helix family that localizes adjacent to the inner nuclear membrane [72]. Because of its interaction with the major nuclear lamina protein NE81, its juxtaposition to the inner nuclear membrane and its unchanging location during the cell cycle, Src1 has attributes of an inner nuclear membrane protein that is involved in nuclear lamina formation. In other species, Src1 is also implicated in nucleolar organization through its ability to stabilize repetitive rDNA sequences [82]. GFP-Src1 and immune-transmission electron microscopy reveal that $\operatorname{Src1}$ is an inner nuclear membrane protein that is tightly linked to the positioning of nucleoli in Dictyostelium [72]. Future work will have to be done to verify the significance of this relationship and whether Src1 fits the description of a true nucleolar protein. Since Src1 retains its localization throughout mitosis, this puts it in a position to serve as part of the reformation points for nucleolar reassembly during telophase (Figure 3).

\section{Dozens of Unconfirmed Nucleolar Proteins}

Considering that human nucleoli appear to contain over 4500 proteins that are involved in a myriad of essential cell functions with many linked to various diseases, studies on the population of nucleolar proteins of Dictyostelium discoideum are still in their infancy. In addition to the proteins discussed above many others, as side issues from studies not directly related to nucleolar structure and function, have been linked to but not yet proven to reside in its nucleolus.

Based on GFP fluorescence images, Meier et al. [83] suggested that RbdB, a nuclear doublestranded RNA binding protein, accumulates in nucleolar foci along with Dicer B, an RNase. However, no experimental validation of this nucleolar localization has been undertaken. What's more GFP-RbdB foci in that publication appear only at a few nucleolar edges as well as at nuclear periphery away from nucleolar regions, suggesting this association may be random. It will be important to determine if actinomycin D treatments will alter these focal locations or if either RbdB or Dicer B have NoLSs that can be deleted to alter any nucleolar association.

Ase1 is a microtubule cross-linking protein with two homologs (A, B) in Dictyostelium [84]. Ase1A shares moderate similarity to the human PRC1 isoform and, similarly, contains two NLSs (320PIEKLKK327, 612PNNKKK1618). During interphase GFP-Ase1A localizes within the dense patches adjacent to the inner nuclear envelope. During mitosis, the protein becomes distributed in the nucleoplasm before localizing within the spindle. The intense and precise localization of Ase1A as a single dot within the interphase nucleolus of Dictyostelium, and its translocations during mitosis, make it a strong candidate as a valid nucleolar protein [84]. Ase1 appears to localize in NoSC2, the nucleolar residence of Snf12, but any colocalization remains to be verified.

A search for nucleolar proteins of Dictyostelium at the Uniprot website (www.uniprot.org) generated a list of over 60 putative nucleolar proteins that were identified as such based on molecular similarity, function or process. For example, multiple low molecular weight ribonucleoproteins and snRNA associated proteins dominated the list. Also included were HEAT-repeat containing proteins, processome components, GTP-binding proteins and others including unknown proteins. Nop56 is another putative nucleolar protein for which antibodies are available online but its nucleolar localization has not been validated. While these proposed constituents support the universality of identified nucleolar functions in Dictyostelium, without further analyses, how they affect its overall structure and function and how they might translocate during mitosis or stress remain to be investigated.

\section{Protein Associates of Nucleolar Proteins}

Studying proteins that interact with nucleolar proteins can provide additional insight into their functions in situ or when they translocate to different cellular locales. For Dictyostelium, this area of research has only just begun. Yeast two hybrid and co-immunoprecipitation analyses revealed 
that NumA1 not only binds to nucleolar Cbp4a but also to nuclear PsaA. PsaA shares the critical domains of human Psa including GAMEN and Zinc-binding domains and, similarly, is also inhibited by bestatin methyl ester (BME) $[60,85]$. DdPsaA has been proven to possess defined NLS and NES sequences. Those studies also revealed the importance of on DdPsaA in both cell proliferation and cell differentiation coinciding with the central functions proposed for NumA1. These tasks were further supported by the finding that DdPsaA in turn binds to cyclin-dependent kinase 5 (DdCdk5) [86]. In support, the Cdk inhibitor roscovitine was effective on DdCdk5 activity and dose-dependently inhibited cell proliferation [86]. Since the understanding of the relationship between nucleolar integrity and cell cycle progression remains to be elucidated in any organism, defining the interplay between NumA1, DdPsaA and DdCdk5 during cell proliferation should be especially enlightening.

\section{Nucleolar Prion-like Proteins}

Proteins possessing prion-like domains (PLDs) are implicated in numerous protein-misfolding diseases, especially neurodegenerative diseases [87]. Enriched in glycine and polar amino acids, PLDs are low complexity amino acid sequences often found in RNA-binding proteins. In the nucleus, these PLD proteins aggregate with other proteins as discrete paraspeckles. Dictyostelium not only has a $\mathrm{Q} / \mathrm{N}$-enriched proteome, it also has the highest amount of prion-like proteins of the organisms studied so far [88]. Overexpression of human huntingtin exon 1 or yeast prion protein Supp35 does not lead to the expected formation of toxic cytosolic aggregates but instead produces harmless, soluble proteins [88]. However, disruption of molecular chaperone function causes these proteins to form insoluble cytotoxic assemblages. Of relevance here is that small accumulations of the huntingtin and yeast prion-like proteins localize to nucleoli suggesting its role in regulating these events in Dictyostelium. Similar huntingtin aggregates have been discovered adjacent to human nucleoli [89]. Other studies on the huntingtin in Dictyostelium that reveal that the mutant protein does not form aggregates while the normal protein is involved in multiple cellular processes including growth, cation homeostasis, cell motility, cell shape, chemotaxis, cell-cell adhesion, cell fate determination and osmoregulation [6]. Understanding how Dictyostelium prevents prion-like protein aggregation, and the role of the nucleolus in this function, could lead to therapies for preventing the formation of toxic plaques found in Huntington, Alzheimer's, Parkinson's and other prion-based neurodegenerative diseases [90].

\section{Conclusions and Questions}

Once considered to be homogeneous structures, the nucleoli of Dictyostelium discoideum display specific protein localizations suggestive of nucleolar compartmentalization. Nucleolar targeting signals have been identified that target proteins to either the whole nucleolus or specific subcompartments within it and, thus, may have biomedical uses. Other studies of nucleolar proteins have aided in the understanding of mitosis in the social amoebozoans revealing that it is a semi-closed rather than closed mitotic event. Despite these relatively unique aspects that offer valuable evolutionary insights into the nucleolus, the presence of nucleolar proteins that share structural and functional similarities to their mammalian counterparts suggests that Dictyostelium can be a useful system for research into nucleolar-related diseases thus adding to its value as a model organism for biomedical research.

Following the translocation of nucleolar proteins during mitosis, when nuclear dissolution/reformation occurs, has led to some interesting discoveries not only about the paths of the proteins but about the nature of Dictyostelium mitosis. For the latter, the apparently intact nuclear envelope becomes permeable revealing that a semi-closed, not closed, type of mitosis is occurring [25]. As might be expected, different nucleolar proteins demonstrate different patterns of dispersal and intracellular localization during mitosis. Whether this has anything to do with a mitotic function that complements their nucleolar roles, remains to be determined. The unique events that occur after treatment with actinomycin D are also interesting. Why do some nucleolar proteins simply 
translocate individually from nucleoli to other cellular locales after actinomycin D treatment while others remain associated in what could be termed a "nucleolar protein export vesicle"?

The nucleolus of Dictyostelium discoideum is linked to various diseases and abnormal cellular states. It is involved in the response to cellular stress and has gained attention in the study of various neurodegenerative and other diseases some of which were addressed above [6,90]. In humans, abnormal nucleolar size, structure and function are associated with neurodegeneration, various cancers and other diseases [20]. As with any research, many questions remain. Dictyostelium discoideum possesses multiple nucleoli that vary in size and number (2-4) during interphase and then decrease in number to a single nucleolus during development. Is there a dominant nucleolus that determines if more are needed during growth then directs their disappearance during development? What are the differences between the multiple vegetative nucleoli and the single developmental nucleolus?

The discovery of Src1 adds to an already interesting developing story. Is this inner nuclear matrix-binding protein the attachment and organization site for nucleolar localization. Its tight association between nucleoli and the inner nuclear membrane plus its persistent localization through the cell cycle implicates it in this function. Whether or not it serves this function, what are the first nucleolar proteins to start nucleolar reformation during telophase? Would it be one from the nucleoplasmic retained nucleolar proteins or one from those that translocated to the cytoplasm during the semi-closed mitosis. Because of their size, the Cbp4 islands that persist in the nucleoplasm could be sites of storage for some nucleolar proteins during mitosis, ready for nuclear reformation during telophase. While there is a clear relationship between nucleolar proteins and the centrosome, what role does the later play in the formation of the former? The answers to these and previous questions could be useful in advancing Dictyostelium's role as a model research organism as well as providing insight into the evolution of eukaryotic nucleoli.

Several nuclear and nucleolar proteins have recently been suggested to be promising targets for anti-cancer drugs [90]. Despite this, the targeting of proteins to the nucleolus is still not fully understood. As focus on the nucleolus increases with the understanding of its importance in a diversity of diseases, understanding the targeting of nucleolar proteins is of key importance since it will serve as a guide to developing vehicles for pharmaceutical delivery. The NLS/NoLS peptides of NumA1 could be of biomedical value since the conjugation of peptides to drugs to target their delivery to specific subcellular locales often results in enhanced efficacy coupled with decreased side effects.

Nuclear localization signals (NLSs), that also act as nucleolar localization signals (NoLSs), have been identified for two nucleolar proteins (i.e., NumA1 and Snf12) in Dictyostelium. These NLS/NoLSs represent the first NoLSs and first NLS/NoLSs identified in this amoebozoan. A perplexing aspect of nucleolar protein localization is how some NLSs, that share sequence similarity to other strict NLS, can also serve as functional NoLSs. It has not been possible in any species to precisely define what the critical and essential attributes of an NoLS are. That said, all four of the NLSs in NumA1 (PKSKKKF, KKSYQDPEIIAHSRPRK, RPRK and PTKKRSL) can localize FITC to nucleoli revealing these sequences are all NoLS/NLS [51,62]. Thus, they potentially could serve as vehicles for general delivery to the nucleolus. In contrast, FITC-KRKR localizes to NoSC2, demonstrating that this NoLS not only localizes to the nucleolus but to a specific region within it. The identification of NLS/NoLS-binding proteins could provide some insight into whether these peptide sequences would be useful for drug delivery in other organisms.

Funding: This work was originally supported by a Natural Sciences \& Engineering Research Council Discovery Grant. Publication costs were paid by the Department of Biology, University of Toronto, Mississauga.

Acknowledgments: Robert A. Huber and Michael A. Myre are thanked for comments on an early draft of this manuscript.

Conflicts of Interest: The author declares no conflict of interest. 


\section{References}

1. O'Day, D.H.; Catalano, A. (Eds.) Proteins of the Nucleolus: Regulation, Translocation and Biomedical Functions; Springer Publishing Company: London, UK, 2013; 371p.

2. Villacís, L.N.; Wong, M.S.; Ferguson, L.L.; Hein, N.; George, A.J.; Hannan, K.M. New roles for the nucleolus in health and disease. BioEssays 2018, 40, 1700233. [CrossRef] [PubMed]

3. Visintin, R.; Amon, A. The nucleolus: The magician's hat for cell cycle tricks. Curr. Opin. Cell Biol. 2000, 12, 372-377. [CrossRef]

4. Pederson, T.; Tsai, R.Y.L. In search of nonribosomal nucleolar protein function and regulation. J. Cell Biol. 2009, 184, 771-776. [CrossRef]

5. Williams, J.G. Dictyostelium finds new roles to model. Genetics 2010, 185, 717-726. [CrossRef]

6. Myre, M.A.; Huber, R.J.; O’Day, D.H. Functional Analysis of Proteins Involved in Neurodegeneration Using the Model Organism Dictyostelium: Alzheimer's, Huntington's and Batten Disease. In Molecular-Genetic and Statistical Techniques for Behavioral and Neural Research; Gerlai, R.T., Ed.; Elsevier: Amsterdam, The Netherlands, 2018; Chapter 16; pp. 491-521.

7. Benichou, J.C.; Quiviger, B.; Ryter, A. Cytochemical study of the nucleolus of the slime-mold Dictyostelium discoideum. J. Ultrastruct. Res. 1983, 84, 60-66. [CrossRef]

8. Maclean, N.; Garside, K.; Bradley, M.C.; Wood, C. The nucleus of axenically grown Dictyostelium discoideum: Studies on its division cycle isolation and conformation. Experientia 1984, 40, 1207-1214. [CrossRef]

9. Roos, U.P.; Bottini, F.; Jenni, V. Morphology of the nucleolus in undifferentiated amebas of Dictyostelium discoideum. Eur. J. Protistol. 1992, 28, 94-101. [CrossRef]

10. Sameshima, M.; Fujimoto, H.; Imai, Y.; Tsukita, S.; Hashimoto, Y. Relation of nucleolar structure and position to the cytoplasmic microtubule system in Dictyostelium. Cell Motil. Cytoskel. 1991, 18, 293-303. [CrossRef]

11. Sameshima, M. The orientation of nucleus, nucleus-associated body and protruding nucleolus in aggregating Dictyostelium discoideum. Exp. Cell Res. 1985, 156, 341-350. [CrossRef]

12. Maeda, Y.; Takeuchi, I. Cell differentiation and fine structures in the development of the cellular slime molds. Dev. Growth Differ. 1969, 11, 232-245. [CrossRef]

13. Simon, I.; Olins, D.E. Higher-order association of extrachromosomal ribosomal RNA genes in Dictyostelium discodeum. Cell Biol. Int. 1994, 18, 1091-1094. [CrossRef] [PubMed]

14. Cockburn, A.F.; Taylor, W.C.; Firtel, R.A. Dictyostelium rDNA consists of non-chromosomal palindromic dimers containing $5 S$ and $36 \mathrm{~S}$ coding regions. Chromosoma 1978, 70, 19-29. [CrossRef] [PubMed]

15. Hohl, H.R.; Miura-Santo, L.Y.; Cotter, D.A. Ultrastructural changes during formation and germination of microcysts in Polysphondylium pallidum, a cellular slime mould. J. Cell Sci. 1970, 7, 285-305. [PubMed]

16. Schaap, P.; Vandermolen, L.; Konijn, T.M. The vacuolar apparatus of the simple cellular slime mold Dictyostelium minutum. Biol. Cell. 1981, 41, 133-142.

17. Kawashima, K.; Sameshima, M.; Izawa, M. Isolation of nucleoli from the cellular slime mold Dictyostelium discoideum, strain A-3. Cell Struct. Funct. 1979, 4, 183-191. [CrossRef]

18. Catalano, A.; O'Day, D.H. Evidence for nucleolar subcompartments in Dictyostelium. Biochem. Biophys. Res. Commun. 2015, 456, 901-907. [CrossRef] [PubMed]

19. Cocucci, S.M.; Sussman, M. RNA in cytoplasmic and nuclear fractions of cellular slime mold amebas. J. Cell Biol. 1970, 45, 399-407. [CrossRef]

20. Farley-Barnes, K.; McCann, K.L.; Ogawa, L.M.; Merkel, J.; Surovtseva, Y.V.; Baserga, S.J. Diverse regulators of human ribosome biogenesis discovered by changes in nucleolar number. Cell Rep. 2018, 22, 1923-1934. [CrossRef]

21. Ishida, S.; Maeda, Y.; Takeuchi, I. Anucleolate mutant of cellular slime mold Dictyostelium discoideum. J. Gen. Microbiol. 1974, 81, 491-499. [CrossRef]

22. Luciani, M.F.; Song, Y.; Sahrane, A.; Kosta, A.; Golstein, P. Early nucleolar disorganization in Dictyostelium cell death. Cell Death Dis. 2017, 8, e2528. [CrossRef]

23. Andersen, J.S.; Lam, Y.W.; Leung, A.K.L.; Ong, S.E.; Lyon, C.E.; Lamond, A.I.; Mann, M. Nucleolar proteome dynamics. Nature 2005, 433, 77-83. [CrossRef] [PubMed]

24. Kitanishi-Yumura, T.; Fukui, Y. Reorganization of microtubules during mitosis in Dictyostelium: Dissociation from MTOC and selective assembly/disassembly in situ. Cell Motil. Cytoskel. 1987, 8, 106-117. [CrossRef] 
25. O'Day, D.H.; Budniak, A. Nucleocytoplasmic Protein Translocation during Mitosis in the Social Amoebozoan Dictyostelium discoideum. Biol. Rev. 2015, 90, 126-141. [CrossRef] [PubMed]

26. Moerman, A.M.; Klein, C. Dictyostelium discoideum Hsp32 is a resident nucleolar heat- shock protein. Chromosoma 1998, 107, 145-154. [CrossRef] [PubMed]

27. Shaw, P.J.; Highett, M.I.; Beven, A.F.; Jordan, E.G. The nucleolar architecture of polymerase I transcription and processing. EMBO J. 1995, 14, 2896-2906. [CrossRef] [PubMed]

28. Grisendi, S.; Mecucci, G.; Falini, B.; Pandolfi, P. Nucleophosmin and cancer. Nat. Rev. Cancer 2006, 6, $493-505$. [CrossRef] [PubMed]

29. Xue, Z.; Melese, T. Nucleolar proteins that bind NLSs: A role in nuclear import or ribosome biogenesis? Trends Cell Biol. 1994, 4, 414-417. [CrossRef]

30. Szebeni, A.; Herrera, J.E.; Olson, M.O.J. Interaction of nucleolar protein B23 with peptides related to nuclear localization signals. Biochemistry 1995, 34, 8037-8042. [CrossRef]

31. Creancier, L.; Prats, H.; Zanibellato, C.; Amalric, F.; Bugler, B. Determination of the functional domains involved in nucleolar targeting of nucleolin. Mol. Biol. Cell 1993, 4, 1239-1250. [CrossRef]

32. Dingwall, C.; Laskey, R.A. Nuclear targeting sequences-A consensus. Trends Biochem. Sci. 1991, 16, 478-481. [CrossRef]

33. Balbo, A.; Bozzaro, S. Cloning of Dictyostelium eIF6 (p27 ${ }^{\mathrm{BBP}}$ ) and mapping its nucle(ol)ar localization subdomains. Eur. J. Cell Biol. 2006, 85, 1069-1078. [CrossRef] [PubMed]

34. Sanvito, F.; Piatti, S.; Villa, A.; Bossi, M.; Lucchini, G.; Marchisio, P.C.; Biffo, S. The $\beta 4$ integrin interactor p27 $7^{\mathrm{BBP} / \mathrm{eIF} 6}$ is an essential nuclear matrix protein involved in $60 \mathrm{~S}$ ribosomal subunit assembly. J. Cell Biol. 1999, 144, 823-837. [CrossRef] [PubMed]

35. Si, K.; Maitra, U. The Saccharomyces cerevisiae homologue of mammalian translation initiation factor 6 does not function as a translation initiation factor. Mol. Cell Biol. 1999, 19, 1416-1426. [CrossRef] [PubMed]

36. Wood, L.C.; Ashby, M.N.; Grunfeld, C.; Feingold, K.R. Cloning of murine translation initiation factor 6 and functional analysis of the homologous sequence YPR016c in Saccharomyces cerevisiae. J. Biol. Chem. 1999, 274, 11653-11659. [CrossRef] [PubMed]

37. Shimarmura, A. Shwachman-Diamond Syndrome. Semin. Hematol. 2006, 43, 178-188. [CrossRef] [PubMed]

38. Ng, C.L.; Waterman, D.G.; Koonin, E.V.; Walters, A.D.; Chong, J.P.J.; Isupov, M.N.; Lebedev, A.A.; Bunka, D.H.J.; Stockley, P.G.; Ortiz-Lombardia, M.; et al. Conformational flexibility and molecular interactions of an archaeal homologue of the Shwachman-Bodian-Diamond syndrome protein. BMC Struct. Biol. 2009, 9, 32. [CrossRef] [PubMed]

39. Weiss, F.; Guidice, E.; Churcher, M.; Jin, L.; Hilcenko, C.; Wong, C.C.; Traynor, D.; Kay, R.R.; Warren, A.J. Mechanism of eIF6 release from the nascent 60s ribosomal subunit. Nature Struct. Molec. Biol. 2016, 22, 914-919. [CrossRef]

40. Scott, M.S.; Troshin, P.V.; Barton, G.J. NoD: A nucleolar localization sequence detector for eukaryotic and viral proteins. BMC Bioinform. 2011, 12, 317. [CrossRef]

41. Sillo, A.; Bloomfield, G.; Balest, A.; Balbo, A.; Pergolizzi, B.; Peracino, B.; Skelton, J.; Ivens, A.; Bozzaro, S. Genome-wide transcriptional changes induced by phagocytosis or growth on bacteria in Dictyostelium. BMC Genom. 2008, 9, 291. [CrossRef]

42. Morita, T.H.; Amagai, A.; Maeda, Y. Unique behavior of a Dictyostelium homologue of TRAP-1, coupling with differentiation of D. discoideum cells. Exp. Cell Res. 2002, 280, 45-54. [CrossRef]

43. Morita, T.H.; Amagai, A.; Maeda, Y. Translocation of the Dictyostelium TRAP1 homologue to mitochondria induces a novel prestarvation response. J. Cell Sci. 2004, 117, 5759-5770. [CrossRef] [PubMed]

44. Morita, T.; Yamaguchi, H.; Amagai, A.; Maeda, Y. Involvement of the TRAP-1 homologue, Dd-TRAP1, in spore differentiation during Dictyostelium development. Exp. Cell Res. 2005, 303, 425-431. [CrossRef] [PubMed]

45. Yamaguchi, H.; Morita, T.; Amagai, A.; Maeda, Y. Changes in spatial and temporal localization of Dictyostelium homologues of TRAP1 and GRP94 revealed by immunoelectron microscopy. Exp. Cell Res. 2005, 303, 415-424. [CrossRef]

46. Francione, L.M.; Annesley, S.J.; Carilla-Latorre, S.; Escalante, R.; Fisher, P.R. The Dictyostelium model for mitochondrial disease. Semin. Cell. Dev. Biol. 2011, 22, 120-130. [CrossRef] [PubMed] 
47. Ohta, Y.; Ohba, T.; Miyamoto, E. Ca ${ }^{2+} /$ calmodulin-dependent protein kinase II: Localization in the interphase nucleus and the mitotic apparatus of mammalian cells. Proc. Natl. Acad. Sci. USA 1990, 87, 5341-5345. [CrossRef] [PubMed]

48. Torgan, C.E.; Daniels, M.P. Calcineurin localization in skeletal muscle offers insights into potential new targets. J. Histochem. Cytochem. 2006, 54, 119-128. [CrossRef] [PubMed]

49. Wong, E.C.; Saffitz, J.E.; McDonald, J.M. Association of calmodulin with isolated nuclei from rat hepatocytes. Biochem. Biophys. Res. Commun. 1991, 181, 1548-1556. [CrossRef]

50. Thorogate, R.; Torok, K. Role of $\mathrm{Ca}^{2+}$ activation and bilobal structure of calmodulin in nuclear and nucleolar localization. Biochem. J. 2007, 402, 71-80. [CrossRef]

51. Myre, M.A.; O’Day, D.H. Nucleomorphin. A novel, acidic, nuclear calmodulin-binding protein from Dictyostelium that regulates nuclear number. J. Biol. Chem. 1974, 277, 19735-19744. [CrossRef]

52. Myre, M.A.; O'Day, D.H. Dictyostelium calcium-binding protein 4a interacts with nucleomorphin, a BRCT-domain protein that regulates nuclear number. Biochem. Biophys. Res. Commun. 2004, 322, 665-671. [CrossRef]

53. Catalano, A.; O'Day, D.H. Calmodulin-binding proteins in the model organism Dictyostelium: A complete and critical review. Cell. Signal. 2008, 20, 277-291. [CrossRef] [PubMed]

54. Sansam, C.L.; Wells, K.S.; Emeson, R.B. Modulation of RNA editing by functional nucleolar sequestration of ADAR2. Proc. Natl. Acad. Sci. USA 2003, 100, 14018-14023. [CrossRef] [PubMed]

55. Lim, M.J.; Wang, X.W. Nucleophosmin and human cancer. Cancer Detect. Prev. 2006, 30, 481-490. [CrossRef] [PubMed]

56. Myre, M.A.; O'Day, D.H. Dictyostelium nucleomorphin is a member of the BRCT- domain family of cell cycle checkpoint proteins. Biochim. Biophys. Acta 2004, 1675, 192-197. [CrossRef] [PubMed]

57. Heath, E.M.; Chan, S.M.; Minden, M.D.; Murphy, T.; Shlush, L.I.; Schimmer, A.D. Biological and clinical consequences of NPM1 in mutations in AML. Leukemia 2017, 31, 798-807. [CrossRef] [PubMed]

58. Box, J.K.; Paquet, N.; Adams, M.N.; Boucher, D.; Bolderson, E.; O’Byrne, K.J.; Richard, D.J. Nucleophosmin: From structure and function to disease development. BMC Mol. Biol. 2016, 17, 19. [CrossRef] [PubMed]

59. Bhutani, N.; Venkatraman, P.; Goldberg, A.L. Puromycin-sensitive aminopeptidase is the major peptidase responsible for digesting polyglutamine sequences released by proteasomes during protein degradation. EMBO J. 2007, 26, 1385-1396. [CrossRef]

60. Catalano, A.; Poloz, Y.; O’Day, D.H. Dictyostelium puromycin-sensitive aminopeptidase A is a nucleoplasmic nucleomorphin-binding protein that relocates to the cytoplasm during mitosis. Histochem. Cell. Biol. 2011, 136, 677-688. [CrossRef]

61. O'Day, D.H.; Poloz, Y.; Myre, M.A. Differentiation inducing factor-1 (DIF-1) induces gene and protein expression of the Dictyostelium nuclear calmodulin-binding protein nucleomorphin. Cell. Signal. 2009, 21, 317-323. [CrossRef]

62. Myre, M.A.; O'Day, D.H. An N-terminal nuclear localization sequence but not the calmodulin-binding domain mediates nuclear localization of nucleomorphin, a protein that regulates nuclear number in Dictyostelium. Biochem. Biophys. Res. Commum. 2005, 332, 157-166. [CrossRef]

63. Russo, G.; Ricciardelli, G.; Pietropaolo, C. Different domains cooperate to target the human ribosomal L7a protein to the nucleus and to the nucleoli. J. Biol. Chem. 1997, 272, 5229-5235. [CrossRef] [PubMed]

64. Collavoli, A.; Hatcher, C.J.; He, J.; Okin, D.; Deo, R.; Basson, C.T. TBX5 nuclear localization is mediated by dual cooperative intramolecular signals. J. Mol. Cell. Cardiol. 2003, 35, 1191-1195. [CrossRef]

65. Theodore, M.; Kawai, Y.; Yang, J.; Kleshchenko, Y.; Reddy, S.P.; Villalta, F.; Arinze, I.J. Multiple nuclear localization signals function in the nuclear import of the transcription factor Nrf2. J. Biol. Chem. 2008, 283, 14176. [CrossRef] [PubMed]

66. Birbach, A.; Bailey, S.T.; Ghosh, S.; Schmid, J.A. Cytosolic, nuclear and nucleolar localization signals determine subcellular distribution and activity of the NF-kB-inducing kinase NIK. J. Cell Sci. 2004, 117, 3615-3624. [CrossRef] [PubMed]

67. Kakuk, A.; Friedlander, E.; Vereb Jr, G.; Lisboa, D.; Bagossi, P.; Toth, G.; Gergely, P.; Vereb, G. Nuclear and nucleolar localization signals and their targeting function in phosphatidylinositol 4-kinase PI4K230. Exp. Cell Res. 2008, 314, 2376-2388. [CrossRef] 
68. Dharamsi, A.; Tessarolo, D.; Coukell, B.; Pun, J. CBP1 associates with the Dictyostelium cytoskeleton and is important for normal cell aggregation under certain developmental conditions. Exp. Cell Res. 2000, 258, 298-309. [CrossRef] [PubMed]

69. Dorywalska, M.; Coukell, B.; Dharamsi, A. Characterization and hetereologous expression of cDNAs encoding two novel closely related $\mathrm{Ca}^{2+}$-binding proteins in Dictyostelium discoideum. Biochim. Biophys. Acta 2000, 356-361. [CrossRef]

70. Sakamoto, H.; Nishio, K.; Tomisako, M.; Kuwayama, H.; Yoshimasa, T.; Suetake, I.; Tajima, S.; Ogihara, S.; Coukell, B.; Maeda, M. Identification and characterization of novel calcium-binding proteins of Dictyostelium and their spatial expression patterns during development. Develop. Growth Differ. 2003, 45, 507-514. [CrossRef]

71. Catalano, A.C.; O’Day, D.H. Rad53 homologue forkhead-associated kinase A (FhkA) and Ca ${ }^{2+}$-binding protein $4 \mathrm{a}(\mathrm{CBP} 4 \mathrm{a})$ are nucleolar proteins that differentially redistribute during mitosis in Dictyostelium. Cell Div. 2013, 8, 4. [CrossRef]

72. Batsios, P.; Ren, X.; Baumann, O.; Larochelle, D.A.; Graf, R. Src1 is a protein of the inner nuclear membrane interacting with the Dictyostelium. Lamin NE81. Cells 2016, 5, 13. [CrossRef]

73. Reisman, D.; Glaros, S.; Thompson, E.A. The SWI/SNF complex and cancer. Oncogene 2009, 28, 1653-1668. [CrossRef]

74. Li, S.; Liu, C.; Li, N.; Hao, T.; Han, T.; Hill, D.E.; Vidal, M.; Lin, J.D. Genome-wide coactivation analysis of PGC-1 $\alpha$ identifies BAF60a as a regulator of hepatic lipid metabolism. Cell Metab. 2008, 8, 105-117. [CrossRef] [PubMed]

75. Catalano, A.C.; O'Day, D.H. Nucleoplasmic/nucleolar translocation and identification of a nuclear localization signal (NLS) in Dictyostelium BAF60a/SMARCD1 homologue Snf12. Histochem. Cell Biol. 2012, 138, 515-530. [CrossRef] [PubMed]

76. Boulon, S.; Westman, B.J.; Hutten, S.; Boisvert, F.-M.; Lamond, A.I. The nucleolus under stress. Mol. Cell 2010, 40, 216-227. [CrossRef]

77. Kodiha, M.; Chu, A.; Lazrak, O.; Stochaj, U. Stress inhibits nucleocytoplasmic shuttling of heat shock protein hsc70. Am. J. Physiol. Cell. Physiol. 2005, 289, C1034-C1041. [CrossRef] [PubMed]

78. Stracker, T.H.; Usui, T.; Petrini, J.H.J. Taking the time to make important decisions: The checkpoint effector kinases Chk1 and Chk2 and the DNA damage response. DNA Repair 2009, 8, 1047-1054. [CrossRef] [PubMed]

79. Smolka, M.B.; Albuquerque, C.P.; Chen, S.-H.; Schmidt, K.H.; Wei, X.X.; Kolodner, R.D.; Zhou, H. Dynamic changes in protein-protein interaction and protein phosphorylation probed with amine-reactive isotope tag. Mol. Cell Proteom. 2005, 4, 1358-1369. [CrossRef] [PubMed]

80. Yu, B.; Fey, P.; Kestin-Pilcher, K.E.; Federov, A.; Prakash, A.; Chisholm, R.L.; Wu, J.Y. Spliceosomal genes in the Dictyostelium genome: A comparison with those in H. sapiens, D. melanogaster, A. thaliana and S. cerevisiae. Protein Cell 2011, 2, 395-409. [CrossRef]

81. Saha, D.; Banerjee, S.; Bashir, S.; Vijayraghavan, U. Context dependent splicing functions of Bud31/Ycr063w define its role in budding and cell cycle progression. Biochem. Biophys. Res. Commun. 2012, 424, 579-585. [CrossRef]

82. Mekhail, K.; Seebacher, J.; Gygi, S.P.; Moazed, D. Role for perinuclear chromosome tethering in maintenance of genome stability. Nature 2008, 456, 667-670. [CrossRef]

83. Meier, D.; Kruse, J.; Buttlar, J.; Friedrich, M.; Zenk, F.; Boesler, B.; Forstner, K.U.; Hammann, C.; Nellen, W. Analysis of the microprocessor in Dictyostelium: The role of Rdb8, a dsRNA binding protein. PLoS Genet. 2016, 12, e1006057. [CrossRef] [PubMed]

84. Tikhonenko, I.; Irizarry, K.; Khodjakov, A.; Koonce, M.P. Organization of microtubule assemblies in Dictyostelium syncytia depends on the microtubule crosslinker, Ase1. Cell. Mol. Life Sci. 2016, 73, 859-868. [CrossRef] [PubMed]

85. Poloz, Y.; Catalano, A.; O'Day, D.H. Bestatin inhibits cell growth, division and differentiation in Dictyostelium through inhibition of puromycin sensitive aminopeptidase. Eukaryot. Cell 2012, 11, 545-557. [CrossRef] [PubMed]

86. Huber, R.J.; O'Day, D.H. Nucleocytoplasmic transfer of cyclin dependent kinase 5 and its binding to puromycin-sensitive aminopeptidase in Dictyostelium discoideum. Histochem. Cell Biol. 2011, 136, 177-189. [CrossRef] [PubMed] 
87. Hennig, S.; Kong, G.; Mannen, T.; Sadowska, A.; Kobelke, S.; Blythe, A.; Knott, G.J.; Iyer, K.S.; Ho, D.; Newcombe, E.A.; et al. Prion-like domains n RNA binding proteins are essential for building subnuclear paraspeckles. J. Cell Biol. 2015, 210, 529-539. [CrossRef] [PubMed]

88. Malinovska, L.; Palm, S.; Gibson, K.; Verbavatz, J.-M.; Alberti, S. Dictyostelium discoideum has a highly Q/N-rich proteome and shows an unusual resilience to protein aggregation. Proc. Natl. Acad. Sci. USA 2015, 112, E2620-E2629. [CrossRef] [PubMed]

89. Lehman, N.L. The ubiquitin proteasome system in neuropathology. Acta Neuropathol. 2009, 118, 329-347. [CrossRef] [PubMed]

90. Mijatovic, T.; De Neve, N.; Gailly, P.; Mathieu, V.; Haibe-Kains, B.; Bontempi, G.; Lapeira, J.; Decaestecker, C.; Facchini, V.; Kiss, R. Nucleolus and c-Myc: Potential targets of cardenolide-mediated antitumor activity. Mol. Cancer Ther. 2008, 7, 1285-1296. [CrossRef]

(C) 2019 by the author. Licensee MDPI, Basel, Switzerland. This article is an open access article distributed under the terms and conditions of the Creative Commons Attribution (CC BY) license (http://creativecommons.org/licenses/by/4.0/). 